\title{
Análise do uso do partograma como instrumento de redução de complicações do parto
}

\author{
Analysis of the use of partogram as instrument to reduce labor complications
}

Análisis del uso de lo partograma como herramienta para reducir las complicaciones del parto

Rubenilson Caldas Valois ${ }^{1 *}$, Antônia Eliene da Silva Oliveira ${ }^{2}$, Deusana Lúcia Sena Batista ${ }^{2}$, Marcileide Silva da Costa $^{2}$, Fernanda Carmo dos Santos ${ }^{2}$, Wanne Thaynara Vaz Gurjão ${ }^{2}$, Jorgeany Soares Parente ${ }^{2}$, Amanda Gonçalves de Souza da Silva ${ }^{1}$, Tamires de Nazaré Soares ${ }^{3}$, Rubia Danielle Caldas Valois ${ }^{3}$, Marcia Helena Machado Nascimento'.

\section{RESUMO}

Objetivo: Analisar o preenchimento do partograma durante a assistência ao trabalho de parto e parto em um hospital público em Belém-Pará. Métodos: Estudo quantitativo com características prospectivas, realizado com 120 partogramas de puérperas atendidas entre fevereiro e abril de 2017 em um hospital público de Belém/Pa. Os dados foram tabulados através do programa Microsoft Excel $2010 \AA$ e analisados no programa Bioestat $5.3 \AA$ para realização de testes descritivos, utilizando-se os testes da média, desvio padrão e Intervalo de Confiança, respeitando-se os padrões éticos da pesquisa. Resultados: Dentre a amostra analisada, 120 $(100,0 \%)$, não estavam preenchidos completamente, assim como, os dados relacionados a pressão arterial, $110(98,0 \%)$ foram os que tiveram maior ausência de registro, juntamente com a variável variedade de posição, encontrada somente em $01(0,01 \%)$. A maior ausência de preenchimento foi evidenciada no eixo 1 do partograma, onde a média de ausência foi de $79,4 \%$ da amostra. Conclusão: Fica evidente que o não preenchimento correto reflete a pouca importância dada a esse instrumento, haja vista que sua não aplicabilidade deixa de proporcionar a parturiente um atendimento seguro, assim como, um melhor acompanhamento das dificuldades que possam surgir durante a evolução do trabalho de parto e parto.

Palavras-chave: Gravidez, Gestantes, Trabalho de parto.

\begin{abstract}
Objective: To analyze the completion of the partogram during labor and delivery care in a public hospital in Belém-Pará. Methods: Quantitative study and with prospective characteristics, with 120 partogram of women between February and April 2017. The data were tabulated through the Microsoft Excel $2010 \AA$ program and analyzed in the Bioestat $5.3 \AA$ program to perform descriptive tests using the test of means and test of standard deviation, respecting the ethical standards of the research. Results: Among the sample analyzed, 120 $(100,0 \%)$ were not fully completed, as well as data related to blood pressure, $110(98,0 \%)$ together with the variable position variety, found only in $01(0,01 \%)$. The greatest absence of filling was evidenced in axis 1 of the partogram, where the mean absence was found na average of 79,4 of the samples. Conclusion: It is evident that the failure to complete correctly reflects the low importance given to this instrument, since its nonapplicability fails to provide the patient with safe care, as well as a better monitoring of the difficulties that may arise during the evolution of labor and childbirth.
\end{abstract}

Keywords: Pregnancy, Pregnant women, Labor obstetric.

\footnotetext{
1 Universidade do Estado do Pará (UEPA), Belém-PA. *E-mail: rubenilsonvalois@gmail.com

2 Centro Universitário Metropolitano da Amazônia (UNIFAMAZ), Belém-PA.

${ }^{3}$ Universidade da Amazônia (UNAMA), Belém-PA.
}

SUBMETIDO EM: $8 / 2019$

ACEITO EM: 9/2019

PUBLICADO EM: 10/2019 


\section{RESUMEN}

Objetivo: Analizar la finalización del partograma durante el parto y la atención en un hospital público en Belém-Pará. Métodos: Estudio cuantitativo y prospectivo con 120 mujeres posparto entre febrero y abril de 2017. Los datos se tabularon usando el programa Microsoft Excel 2010® y se analizaron usando el programa Bioestat $5.3 \AA$ para realizar pruebas descriptivas, usando las pruebas de media y desviación estándar, respetando los estándares éticos de la investigación. Resultados: Entre la muestra analizada, 120 (100,0\%) no se llenaron por completo, ya que los datos relacionados con la presión arterial, $110(98,0 \%)$ tuvieron la mayor ausencia de registro, junto con la variedad de posición variable, encontrado solo en $01(0,01 \%)$. La mayor ausencia de llenado se evidenció en el eje 1 del partograma, donde la ausencia promedio se encontró um promedio de 79,4 de las muestras. Conclusión: Es evidente que el cumplimiento incorrecto refleja la poca importancia dada a este instrumento, ya que su no aplicabilidad deja de proporcionar al parturienta una atención segura, así como un mejor seguimiento de las dificultades que pueden surgir durante la evolución del trabajo de parto y parto.

Palabras clave: Embarazo, Mujeres embarazadas, Trabajo de parto.

\section{INTRODUÇÃO}

A gravidez e o parto são momentos únicos na vida de uma mulher, marcados por intensos sentimentos e emoções. Essas vivências permanecerão na sua memória ao longo da vida, em respeito a esse momento, todos os envolvidos na assistência a essa gestante deverão proporcionar um atendimento humanizado desde o pré-natal até o parto (ANDRADE MAC, LIMA JBMC, 2014).

O parto normal é um evento com características fisiológicas, relacionado ao desenvolvimento de contrações uterinas rítmicas e dolorosas, que auxiliam na dilatação da cérvice uterina, variando de dois a dez centímetros de dilatação, caracterizando uma abertura necessária para a expulsão do concepto (COSTA NM, et al., 2011).

Conceitua-se como distocia uma progressão demasiadamente lenta do trabalho de parto relacionadas à presença de características patológicas que possam interferir na dilatação do colo uterino e com a progressão do concepto através da pelve materna, dificultando desta forma sua expulsão (GRAÇA LM, 2010).

Paula LG, et al. (2000), relata que Emanuel Friedman, em 1951, após minucioso estudo da dilatação do colo uterino ao longo do trabalho de parto, estabeleceu uma curva padrão de normalidade, onde dividiu o trabalho de parto em duas fases: fase ativa e fase latente. A análise gráfica e estatística do trabalho de parto de Friedman permitiu a transformação da avaliação subjetiva em uma possibilidade preditiva, quando após extenso estudo da dilatação da cérvice uterina no trabalho de parto estabeleceu uma curva padrão de normalidade, amplamente utilizada até hoje.

O trabalho de parto e o parto podem ser representados graficamente por um instrumento denominado partograma, utiliza-se para isto linhas denominadas de alerta e de ação, registrando o progresso do trabalho de parto e também servindo como registro documental do mesmo, tudo isso com o objetivo de identificar possíveis alterações, desta forma, diagnosticar distocias, possibilitando uma intervenção precoce, auxiliando em uma tomada de conduta adequada pela equipe obstétrica assistente (TRAVERZIM MAS, NOVARETTI MCZ, 2014).

Identificada a distocia no partograma e reconhecida sua etiologia, a orientação terapêutica poderá ser lógica e eficaz. Sem dúvida, sua utilização melhora a qualidade da assistência clínica ao parto, devendo ser incluída como rotina nas maternidades (BRASIL, 2006).

A Resolução Normativa no 368 dispõe sobre novas regras referentes a parto na saúde suplementar, entre outros, estabelece normas para a correta utilização do partograma, tal como as informações que devem ser prestadas a gestante. Portanto, auxilia com orientações valorosas para que a mulher tome, em comum acordo com o médico assistente, a melhor decisão sobre o parto (BRASIL, 2015).

O partograma pode ter grande utilidade como instrumento para avaliar o processo de parturição de forma 
global, verificando a dilatação do colo; a descida do feto pelo trajeto pélvico; a estática fetal, assim como o batimento cardíaco fetal e as contrações do útero. A avaliação completa, por parte do profissional assistente, de todas essas informações permite o reconhecimento dos principais fatores causadores de evolução normal e anormal do trabalho de parto (VASCONCELOS KL, et al., 2013).

No que se refere aos registros e a passagem de plantão entre as equipes Rocha IMS, et al. (2009), afirmam que o partograma também é considerado um instrumento de comunicação e facilita o conhecimento rápido e imediato do transcorrer do trabalho de parto. Nele também deve ser registrado o monitoramento cardíaco fetal, o aspecto do líquido amniótico, a dinâmica uterina, entre outros itens importantes.

$\mathrm{Na}$ assistência ao trabalho de parto, o uso adequado do partograma é uma prática que possui valor acessível e demanda treinamento de baixa complexidade, demonstrando ser um instrumento simples e barato, mas que pode ter um grande impacto benéfico, especialmente nessa transição de modelos de assistência obstétrica (GATS, 2015).

Este instrumento pode ser visto também como uma ferramenta de incentivo à documentação adequada do trabalho de parto, que pode auxiliar na tomada de decisões e reduzir a taxa de intervenções e cirurgias desnecessárias, onde hoje no Brasil a cesariana representa a principal forma de nascimento, chegando a $55,0 \%$ dos partos e em assustadores $84,6 \%$ nos hospitais e maternidades privadas. No serviço público, atualmente a taxa chega próximo aos 40,0\% (BRASIL, 2016).

É extremamente relevante a confirmação do preenchimento correto do partograma, onde os registros devem estar de acordo com as reais informações obtidas no exame obstétrico, descrevendo as condutas realizadas. A qualidade da assistência que se presta a gestante em trabalho de parto, não se resume apenas à existência do partograma, mas também ao seu correto preenchimento, evitando desta forma, intervenções desnecessárias (GIGLIO MRP, et al., 2011).

Segundo Rocha IMS, et al. (2009) o partograma é um instrumento com função de nortear os profissionais durante 0 trabalho de parto quanto à realização das intervenções obstétricas. Porto AMF, et al. (2010) sinalizam que a aplicação deste instrumento de acompanhamento do parto em países de baixa renda contribuiu para o decréscimo na taxa de partos cesáreos.

O Escore de Bologna é utilizado para a observação quantitativa e também avaliação da qualidade assistencial prestada durante o parto normal e nascimento, buscando-se avaliar as melhores condutas e intervenções nos serviços de obstetrícia, de acordo com as evidências científicas mais recentes, dentre estas práticas, encontra-se a aplicação do partograma, como estratégia para avaliar a qualidade dessa assistência (GIGLIO MRP, et al., 2011).

Frente ao exposto, o objeto do estudo foi a utilização do partograma como instrumento na assistência obstétrica e o objetivo foi analisar o preenchimento do partograma durante a assistência ao trabalho de parto e parto em um hospital público em Belém-Pará.

\section{MÉTODOS}

O estudo teve uma abordagem quantitativa descritiva e documental, e com características prospectivas, a fonte de informações foram 120 partogramas contidos em prontuários de puérperas atendidas em fase ativa do trabalho de parto em uma maternidade pública de um hospital regional localizado na cidade de Belém no estado do Pará.

A referida maternidade trabalha com o intuito de oferecer as suas usuárias um atendimento digno e humanizado, e usa o partograma nos moldes recomendados pela diretriz do Ministério da Saúde.

Constituíram-se como amostra deste estudo somente partogramas de prontuários de puérperas que tiveram seu parto iniciado e concluído no referido hospital, no período de fevereiro a abril de 2017, foram excluídas do estudo partogramas de puérperas admitidas em período expulsivo do trabalho de parto, prontuários de puérperas que foram submetidas a parto operatório eletivo, assim como, prontuários de pacientes submetidas à curetagem e aspiração manual intrauterina (AMIU). 
O estudo foi avaliado e aprovado pelo Comitê de Ética em Pesquisa da Faculdade Metropolitana da Amazônia-FAMAZ sob o parecer de no 1.999.002, CAAE: 66007717.2.0000.5701, onde também recebeu aprovação do Setor de Ensino e Pesquisa (SEP) do hospital regional Dr. Abelardo Santos, através de um Termo de Consentimento de Utilização de Dados destinado à direção do hospital.

A coleta de dados foi realizada entre os meses de fevereiro e abril de 2017, no período da manhã, mediante autorização da enfermeira responsável pelo setor. As informações foram obtidas através da aplicação de formulários, estes foram elaborados dividindo as variáveis analisadas em três eixos para melhor avaliação e análise, contendo perguntas fechadas sobre o correto preenchimento e aplicabilidade do partograma, com o objetivo de identificar, analisar e quantificar os fatores mais incidentes no manuseio do partograma. Não houve contato direto com as pacientes, os formulários foram identificados através de códigos numéricos sequenciais $(01,02, \ldots, 119,120)$.

A análise das informações coletadas foi elaborada através de recursos do programa Microsoft Excel $2010 \AA$, onde foi realizada tabulação dos dados em planilhas para processamento e descrição dos resultados, também foi utilizado o programa Bioestat $5.3 \AA$ para realização de testes descritivos (média, desvio padrão e Intervalo de Confiança).

O desenvolvimento do estudo ocorreu dentro dos padrões éticos da pesquisa, onde foram considerados todos os aspectos éticos previstos na Resolução no 466/2012 do Conselho Nacional de Saúde, levando em consideração as diretrizes e normas regulamentadoras de pesquisa, incorporando as quatro referências básicas da bioética, envolvendo a relevância social da pesquisa com vantagens significativas para os sujeitos do estudo (BRASIL, 2012).

\section{RESULTADOS E DISCUSSÃO}

De acordo com os dados evidenciados na tabela 1, verificou-se que as maiores incidências de não preenchimentos foram encontradas nas variáveis: hora, data provável do parto (DPP) e grupo sanguíneo, com 100 (83,3\%), 97 (80,2\%) e 118 (98,3\%), respectivamente. Encontrando-se uma média de 40,6 (IC 95 $\% 36,8 \leq \mu \leq 44,4$ ) de itens preenchidos em contrapartida, 79,4 (IC $95 \% 75,6 \leq \mu \leq 83,2$ ) das variáveis não estavam preenchidos, com desvio padrão de 20,8 nos partogramas analisados (Tabela 1).

Tabela 1 - Distribuição dos itens avaliados do eixo 1 dos partogramas avaliados de acordo com 0 preenchimento no período de fevereiro à abril de 2017, Belém, PA.

\begin{tabular}{|c|c|c|c|c|}
\hline \multirow[t]{2}{*}{ Itens avaliados } & \multicolumn{2}{|c|}{ Sim } & \multicolumn{2}{|c|}{ Não } \\
\hline & $\mathbf{N}$ & $\%$ & $\mathbf{N}$ & $\%$ \\
\hline Nome & 76 & 63,3 & 44 & 36,7 \\
\hline Idade & 46 & 38,3 & 74 & 61,7 \\
\hline Leito & 30 & 25,0 & 90 & 75,0 \\
\hline Hora & 20 & 16,7 & 100 & 83,3 \\
\hline Número de gestações & 67 & 55,8 & 53 & 44,2 \\
\hline Número de partos normais & 52 & 43,3 & 68 & 56,7 \\
\hline Número de partos cesarianos & 34 & 28,3 & 86 & 71,7 \\
\hline Número de partos a fórceps & 31 & 25,8 & 89 & 74,2 \\
\hline Número de abortos & 37 & 30,8 & 83 & 69,2 \\
\hline Data da última menstruação (DUM) & 27 & 22,5 & 93 & 77,5 \\
\hline Data provável do parto (DPP) & 23 & 19,2 & 97 & 80,2 \\
\hline Grupo sanguíneo & 02 & 1,7 & 118 & 98,3 \\
\hline Situação & 61 & 50,8 & 59 & 49,2 \\
\hline Apresentação & 62 & 51,7 & 58 & 48,3 \\
\hline Média $(\mu)$ & \multicolumn{2}{|c|}{40,6} & \multicolumn{2}{|c|}{79,4} \\
\hline Desvio padrão & \multicolumn{2}{|c|}{20,8} & & \\
\hline Intervalo de Confiança (IC) 95\% & & & \multicolumn{2}{|c|}{$75,6 \leq \mu \leq 83,2$} \\
\hline
\end{tabular}

Fonte: Valois RC, Oliveira AES, Batista DLS, et al., 2017. 
A não observância do preenchimento desses itens de alguma forma pode incorrer em prejuízo na assistência, além de que desconsiderar o estipulado pelo Ministério da Saúde, através da Portaria no 1.820, de 13 de agosto de 2009, que diz "É direito da pessoa ter atendimento adequado, com qualidade, no tempo certo e com garantia de continuidade do tratamento".

Segundo Rosa E (2014) a data provável do parto (DPP) é a data onde possivelmente ocorrerá o parto, sendo prevista a partir da data da última menstruação (DUM) ou através da primeira ultrassonografia (USG) feita pela gestante, a DUM auxilia na estimativa da idade gestacional.

A análise da amostra evidenciou que o não preenchimento de $97(80,2 \%)$ do item destinado à data provável do parto, assim como o não preenchimento de $93(77,5 \%)$ destinados à data da última menstruação, contribui para possíveis erros de cálculo, podendo com isso no caso de prematuridade colocar em risco a vida do recém-nascido, haja vista que, a equipe que está prestando assistência utilizará as informações contidas no partograma.

Com base nestas informações, pode-se afirmar que tais variáveis não têm recebido a devida importância dos profissionais de saúde para o seu correto preenchimento, assim como desconsiderando o histórico das gestações anteriores comprometendo a qualidade da assistência, atestando com isso uma prática de cuidados insegura, sem conformidade com a legislação e código de ética (GATS, 2015).

O item destinado ao grupo sanguíneo, no total da amostra da tabela 1, foi o que apresentou o maior número de ausência de registro, com 118 (98,3\%), dificultando uma segura intervenção no caso de uma possível transfusão, onde Souza GF (2012) afirma que para evitar possíveis danos os registros de enfermagem são elementos imprescindíveis ao cuidado do paciente.

O Conselho Federal de Medicina através da Resolução n.. 1.638/02 define que o prontuário é documento único, de caráter legal, sigiloso e cientifico constituído das principais informações e registros dos clientes, informações estas sobre a saúde do paciente e dos cuidados prestados a este, permitindo desta forma a facilitação da comunicação entre profissionais da equipe assistente e possibilitando continuidade do cuidado ao indivíduo. Esta definição pode ser aplicada ao partograma, haja vista que é parte integrante do prontuário da gestante. Dessa forma, quando não identificado e preenchido corretamente compromete a legalidade da documentação, colaborando para o extravio e perda dos mesmos.

A tabela 2 apresentou uma média de 72,6 (IC95\% $66.8 \leq \mu \leq 78.4$ ) de variáveis não preenchidos e somente uma média de 47,4 (IC95\% $41.6 \leq \mu \leq 53.2$ ) estavam preenchidos corretamente, assim apresentando um desvio padrão de 31,9. O estudo mostrou que as variáveis com maior incidência de não preenchimento foram: variedade de posição, linha de alerta e linha de ação, com 119 (99,2\%), 100 (83,3\%) e 100 (83,3\%) respectivamente (Tabela 2 ).

Tabela 2 - Distribuição dos itens avaliados do eixo 2 dos partogramas avaliados de acordo com o preenchimento no período de fevereiro à abril de 2017, Belém, PA.

\begin{tabular}{|c|c|c|c|c|}
\hline \multirow[t]{2}{*}{ Itens avaliados } & \multicolumn{2}{|c|}{ Sim } & \multicolumn{2}{|c|}{ Não } \\
\hline & $\mathbf{N}$ & $\%$ & $\mathbf{N}$ & $\%$ \\
\hline Data & 59 & 49,2 & 61 & 50,8 \\
\hline Horário & 58 & 48,3 & 62 & 51,7 \\
\hline Avaliação 2/2 h & 46 & 38,3 & 74 & 61,7 \\
\hline Dilatação & 91 & 75,8 & 29 & 24,2 \\
\hline Descida da apresentação & 84 & 70,0 & 36 & 30,0 \\
\hline Variedade da posição & 01 & 0,8 & 119 & 99,2 \\
\hline Linha de alerta & 20 & 16,7 & 100 & 83,3 \\
\hline Linha de ação & 20 & 16,7 & 100 & 83,3 \\
\hline Média $(\mu)$ & \multicolumn{2}{|c|}{47,4} & \multicolumn{2}{|c|}{72,6} \\
\hline Desvio padrão & \multicolumn{2}{|c|}{31,9} & \multirow{2}{*}{\multicolumn{2}{|c|}{$66,8 \leq \mu \leq 78,4$}} \\
\hline Intervalo de Confiança (IC) $95 \%$ & & & & \\
\hline
\end{tabular}

Fonte: Valois RC, Oliveira AES, Batista DLS, et al., 2017. 
A não observação de parâmetros no partograma pode levar a um comprometimento na assistência a parturição, assim como comprometer possíveis intervenções necessárias, sendo de extrema relevância a correta e completo preenchimento do instrumento, pois permitem a prevê uma possível evolução anormal do trabalho de parto, identificando o surgimento de complicações.

A variável dilatação foi preenchida corretamente em 91 (75,8\%), e descida da apresentação preenchida em $84(70,0 \%)$ da amostra, sendo fundamental o correto monitoramento para uma maior segurança durante a evolução do trabalho de parto, conduzindo a equipe que está prestando assistência.

Neme B (2005) refere-se à dilatação cervical como o processo que inclui o esvaecimento cervical e a cérvico dilatação, no qual o colo do útero se amplia de forma gradual, participando da formação do trajeto do parto, permitindo assim, a passagem da parte do feto que se apresenta.

Para Silva LB (2014) a avaliação da dilatação do colo uterino pelo toque vaginal é fundamental para observação da evolução do trabalho de parto, permitindo desta forma, definir toda estática do feto, além de permitir avaliar as características peculiares do colo uterino ao longo do trabalho de parto. No entanto, conforme Pereira WR (2000) este exame em muitas situações realizado unicamente com o objetivo de verificar apenas a dilatação, não é bem aceito pelas mulheres, principalmente, pelo desconforto físico e emocional associado a este procedimento.

Considera-se que a observação destas variáveis contribui para a condução do trabalho de parto seguro, prevenindo riscos de complicações que podem resultar em intervenções inoportunas, ou ainda incorrer em um desfecho de óbito materno e/ou fetal (BRASIL, 2016).

O partograma pode ser um instrumento indicador de qualidade no processo assistencial, a utilização desta ferramenta no acompanhamento do parto auxilia na avaliação da qualidade da assistência prestada a parturiente. Desta forma, contribuindo sobremaneira para o fornecimento de uma visão global do trabalho de parto, o uso deste instrumento torna possível ao profissional que assiste o parto, identificar possíveis complicações no bem-estar da parturiente e do seu feto, assim como a evolução do parto (BHUTTA ZA, et al., 2010).

Quanto ao eixo 3 observado na tabela 3, as variáveis com maior incidência de não preenchimento foram: pressão arterial e uso de medicamentos com $110(91,7 \%)$ e $85(70,8 \%)$ respectivamente, tendo como média de itens preenchimentos de 63,5 (IC95\% $58,6 \leq \mu \leq 68,7$ ) e já o de não preenchimento uma média de 56,5 (IC95\% 51,3 $\leq \mu \leq 61,7$ ) e desvio padrão de 29,0 (Tabela 3).

Tabela 3 - Distribuição dos itens avaliados do eixo 3 dos partogramas avaliados de acordo com 0 preenchimento no período de fevereiro à abril de 2017, Belém, PA.

\begin{tabular}{lcccc}
\hline \multicolumn{1}{c}{ Itens avaliados } & Sim & \multicolumn{2}{c}{ Não } \\
\hline & $\mathbf{N}$ & $\%$ & $\mathbf{N}$ & $\%$ \\
Batimento cardíaco fetal (BCF) & 89 & 74,2 & 31 & 25,8 \\
Dinâmica uterina (DU) & 86 & 71,7 & 34 & 28,3 \\
Avaliação da bolsa amniótica & 78 & 65,0 & 42 & 35,0 \\
Líquido amniótico & 61 & 50,8 & 59 & 49,2 \\
Pressão arterial (PA) & 10 & 8,3 & 110 & 91,7 \\
Uso de medicamentos & 35 & 29,2 & 85 & 70,8 \\
Assinatura & 92 & 76,7 & 28 & 23,3 \\
Diagnóstico final & 57 & 47,5 & 63 & 52,5 \\
\hline Média ( $\boldsymbol{\mu}$ ) & \multicolumn{2}{c}{63,5} & \multicolumn{2}{c}{56,5} \\
Desvio padrão & \multicolumn{2}{c}{59,0} \\
Intervalo de Confiança (IC) $95 \%$ & $58,6 \leq \mu \leq 68,7$ & & $51,3 \leq \mu \leq 1,7$ \\
\hline
\end{tabular}

Fonte: Valois RC, Oliveira AES, Batista DLS, et al., 2017.

Oliveira DLLC, et al. (2014) enfatiza que a hipertensão arterial faz parte das três principais causas de eventos com maior índice de mortalidade relacionas aos partos no Brasil, e ainda, o Ministério da Saúde aponta a hipertensão como a maior causa de morte materna no país (BRASIL, 2012). 
A síndrome hipertensiva específica da gestação é uma das principais patologias que causam morbimortalidade materna e perinatal, associada a uma alta taxa de incidência e de prevalência no Brasil, atualmente ocupa o primeiro lugar dentre as patologias que afetam a mulher no ciclo gravídico e no puerpério, sendo a principal causa de morte em mulheres gestantes, isso ocorrendo, principalmente, quando a mesma evolui para suas formas mais graves, tal como a eclampsia e complicações caracterizadas por hemólise, enzimas hepáticas elevadas e baixa contagem de plaquetas (AGUIAR LRS, et al., 2014).

Os itens avaliados na tabela 3 foram os que mais receberam atenção no preenchimento adequado, demonstrando o cuidado dos profissionais com alguns aspectos no acompanhamento do trabalho de parto, mesmo que em outros itens tenham seu preenchimento inadequado. Fato este evidenciado no item batimento cardíaco fetal (BCF), onde $89(74,2 \%)$ partogramas tiveram os valores preenchidos completamente. De acordo com Rezende J (2008) a correta identificação de alterações no padrão da frequência do coração fetal leva a inferir possibilidades de sofrimento do feto intra-útero, esta técnica propedêutica é utilizada na prática clínica de forma inquestionável.

Para Porto AMF, et al. (2010) o acompanhamento da vitalidade fetal deve feita por meio da ausculta contínua, sendo naquelas mulheres classificadas como de parto com risco habitual poderá ser feito em um intervalo de 15 a 30 minutos na fase de dilatação do colo e a cada 5 minutos na fase de expulsão do feto, levando em consideração as diretrizes para um parto seguro proposto pelo Ministério da Saúde do Brasil, no qual orienta que a frequência cardíaca fetal normalmente varia entre 120 a 160 batimentos a cada minuto (BRASIL, 2013).

A verificação do líquido amniótico foi encontrada somente em 61 (50,8\%) dos partogramas, ou seja, a ausência desse registro compromete o cuidado quando há ocorrências, considerando que a aspiração do líquido amniótico com presença de mecônio pode levar ao desenvolvimento de pneumonia aspirativa, necessidade de ventilação mecânica e morte (DUDELL GD, STOLL BJ, 2009).

Cloherty JP, et al. (2010), afirmam que, a eliminação de mecônio intrauterino em neonatos a termo está relacionada com o aumento do risco de mortalidade perinatal e neonatal, requerendo intervenções ou até administração de oxigênio.

A verificação da condição da bolsa amniótica em $78(65 \%)$ da amostra, ainda é pequena se considerarmos que é um item de extrema importância, pois a perda de líquido amniótico antes do trabalho de parto, independentemente da idade gestacional, entre 30 a $40 \%$ dos casos, é considerada causa principal de partos prematuros, sendo responsável por $20 \%$ dos óbitos perinatais, assim como, é recomendado um cuidado maior no caso de rotura prematura de membranas pré-termo devido ao elevado risco de prolapso do cordão umbilical e consequentemente sofrimento fetal (GOLINO PS, et al. 2006; CAMPOS DA, et al. 2008).

O item destinado à administração de medicamentos não foi preenchido em 85 (70,8\%) dos partogramas analisados, com isso deixando de associar caso ocorresse com as complicações mais frequentes dos fármacos utilizados na indução de trabalho de parto que são a taquissistolia uterina, alterações da frequência cardíaca fetal, falência de indução do trabalho de parto com consequente aumento da taxa de cesariana e a rotura uterina (TEIXEIRA LRM, 2010).

O parâmetro destinado à assinatura encontrado em $92(76,7 \%)$ respeita o que defende Françolin L, et al. (2012), onde refere que, para serem consideradas de qualidade, as anotações contidas no prontuário do paciente, devem conter registros precisos, adequados, atuais e, principalmente legíveis, sem rasuras e com carimbo e assinatura que permita identificar o profissional.

Do total de partogramas analisados no estudo, nenhum teve seu preenchimento de acordo com as recomendações da Organização Mundial de Saúde, o que demostra um descaso com a utilização correta deste instrumento por parte dos profissionais que assistem o trabalho de parto, observou-se que o uso deste instrumento durante a assistência ao parto, não é frequentemente utilizado, e quando se usa, não se preenche de forma adequada, com altos índices de equívocos, elevando o número de intervenções desnecessárias e risco na assistência prestada. 
Para Rocha IMS, et al. (2009) o uso do partograma pode ser considerado uma ótima estratégia que norteará a melhor conduta a ser tomada pelo profissional na ocorrência de distocias, além de ser um ótimo elemento na prática de ensino em hospitais que atuam na formação de novos profissionais, favorecendo desta forma, que futuros profissionais da área obstétrica percebam mais facilmente potenciais complicações no transcurso da assistência ao trabalho de parto.

De acordo com Carvalho IS e Brito RS, (2016) há necessidade de modificar o modelo assistencial obstétrico atual, necessitando reconsiderar práticas intervencionistas na atenção obstétrica, em especial, naquelas como a utilização de instrumentos, tais como o partograma. Esperando-se uma melhoria na assistência obstétrica que seja norteada por práticas baseadas em evidências cientificas.

Os profissionais que atuam na assistência obstétrica devem entender que há necessidade real em se sensibilizar para utilização do partograma durante o acompanhamento do trabalho de parto e parto, e efetivamente começarem a utilizar este instrumento, independentemente de sua obrigatoriedade. Além disso os gestores devem inserir em seus planejamentos capacitações que objetivem a sensibilização para a utilização correta do partograma, alcançando os índices de utilização preconizado pela Organização Mundial de Saúde (BARROS LA, VERÍSSIMO RCSS, 2011).

O partograma mesmo recomendado pela Organização Mundial de Saúde desde 1994, tem sua utilização ainda muito reduzida nas maternidades e Centro de Partos Normais. E com as mudanças assistenciais advindas de nova evidências cientificas, os profissionais mesmo tendo o instrumento como guia de sua assistência, ainda decidem pela não intervenção em situações preconizadas tais como a fase ativa prolongada. Apesar disto, o partograma ainda se demonstra um ótimo instrumento que possibilita nortear as ações de intervenções no trabalho de parto (ROCHA IMS, et al., 2014).

Desta forma, é necessária e essencial uma maior discussão da temática buscando sempre a valorização do parto sem intervenções e que os profissionais estejam qualificados para prestar uma assistência cada vez mais humana e eficiente, reduzindo por consequência as intervenções desnecessárias.

\section{CONCLUSÃO}

Compreende-se existir uma elevada importância na correta utilização do partograma por profissionais que assistem o parto, assegurando as parturientes um atendimento seguro e uma redução de risco de mortes perinatais e de partos anormalmente prolongados. Quanto às dificuldades de aplicabilidade sugere-se capacitações visando o aperfeiçoamento do profissional, tanto para esclarecimentos de dúvidas, quanto para conscientização da importância do instrumento. Para amenizar a ausência de registro no partograma, há necessidade de reconhecimento que esse instrumento é parte integrante do processo para um parto seguro, porém, é necessário que haja uma profunda conscientização quanto ao porquê de estar preenchendo adequadamente, desenvolvendo um compromisso com relação à importância e relevância deste documento.

\section{REFERÊNCIAS}

1. AGUIAR LRS, et al. Análise de estudos sobre as condutas de enfermagem no cuidado a gestante com doença hipertensiva. Rev. Interdi, 2014; 7 (1): 204-215.

2. ANDRADE MAC, LIMA JBMC. O modelo obstétrico que defendemos e com o qual trabalhamos. In: MINISTÉRIO DA SAÚDE. Caderno Humaniza SUS: humanização do parto e do nascimento. Brasília: Editora do Ministério da Saúde, 2014, 465 p. (Caderno Humaniza SUS, v. 4), bloco 1: 19-46.

3. BHUTTA ZA, et al. Countdown to 2015 decade report (2000-10): taking stock of maternal, newborn, and child survival. The lancet. 2010, 375(9730): 2032-2044.

4. BRASIL. Ministério da Saúde. Secretaria de Atenção à Saúde. Departamento de Ações Programáticas Estratégicas. Gestação de alto risco: manual técnico / Ministério da Saúde, Secretaria de Atenção à Saúde, Departamento de Ações Programáticas Estratégicas. 5 ed. Brasília, 2012: Editora do Ministério da Saúde,

5. Parto, Aborto e Puerpério: assistência humanizada à mulher. Brasília (Brasil): Ministério da Saúde, 2006. 199 p.

6. Agência Nacional de Saúde Suplementar. Resolução Normativa n. 368, de 06 de janeiro de 2015.

7. Conselho Nacional de Saúde. Resolução no 466 de 12 de dezembro de 2012. Aprova as diretrizes e normas regulamentadoras de pesquisas envolvendo seres humanos. Diário Oficial da União, Brasília, 13 jun. 2013. p. 59. 
8. Secretaria de Atenção à Saúde Departamento de Atenção Básica, 2013.

9. Ministério da Saúde. Secretaria de atenção à Saúde. Departamento de ações programáticas estratégicas. Área técnica de saúde da mulher. pré-natal e puerpério: atenção qualificada e humanizada - manual técnico. Brasília: Editora do Ministério da Saúde, 2005, 163 p. (Série A. Normas e Manuais Técnicos) - (Série Direitos Sexuais e Direitos Reprodutivos - Caderno oㅜ 5).

10. Ministério da Saúde do brasil. Secretaria de atenção à saúde. Departamento de atenção básica. Atenção ao prénatal de baixo risco. Brasília: editora do ministério da saúde, 2015, 318 p. (cadernos de Atenção Básica, n. 32).

11. CAMPOS DA, et al. Protocolos de Medicina materno-fetal. $2^{a}$ edição. 2008. Lisboa: Lidel,. ISBN 978-972-757-4674.

12. CONSELHO FEDERAL DE MEDICINA. Resolução n. 1638, de 09 de agosto de 2002 - Define prontuário médico e torna obrigatória a criação da Comissão de Revisão de Prontuários nas instituições de saúde.

13. COSTA NM. Episiotomia nos partos normais: uma revisão de literatura. 9. ed.. Mossoró/RN. Revista Facene/Famene, 2011, 9(2): 45-50.

14. CLOHERTY JP, et al. Manual de neonatologia. 6 ed. Rio de Janeiro (RJ): Guanabara Koogan, 2010.

15. DUDELL GD, STOLL BJ. Doenças do Trato Respiratório. In: BEHRMAN, R.E.; KLIEGMAN, R.M.; et al. Nelson: Tratado de Pediatria. 18 ${ }^{\mathrm{a}}$ ed. Rio de Janeiro: Elsevier, 2009. 728-752.

16. FRANÇOLIN L, BRITO MFP. GABRIEL, C.S.; MONTEIRO, T.S.; BERNARDES, A. A. Qualidade dos registros de enfermagem em prontuários de pacientes hospitalizados. Rev. enferm. UERJ, 2012.

17. FRIEDMAN EA. Evolution of graphic analysis of labor. Am J Obstet Gynecol. 1978.

18. GATS (Grupo de Avaliação de Tecnologias em Saúde). Revisando o uso do partograma e seu papel nas indicações de cesariana intraparto. Centro de inovações. Belo Horizonte, 2015.

19. GIGLIO MRP, et al. Avaliação da qualidade da assistência ao parto normal. Revista Brasileira de Ginecologia e Obstetrícia, Rio de Janeiro, oct. 2011, 33(10).

20. GIL, Antônio Carlos. Métodos e técnicas de pesquisa social. 6 ed. São Paulo: Atlas, 2011.

21. GOLINO OS, et al. Rutura Prematura de Membranas: Fisiopatologia, Diagnóstico e Conduta. Femina. Universidade Federal do Maranhão. Out. 2006, 34(10): 711-717.

22. GRAÇA LM. Medicina Materno Fetal. 4ª edição. Lisboa: LIDEL, 2010. ISBN 978-972-757-654-8.

23. NEME B. Obstetrícia básica. São Paulo: Sarvier, 2005, 124-95.

24. OLIVEIRA DLLC, et al. Atenção materna e infantil e marcadores socioculturais. In: Caderno Humaniza SUS. Humanização do parto e do nascimento. Brasília: Editora do Ministério da Saúde, 2014, 465 p. (Caderno Humaniza SUS, v. 4), bloco 1: 48-58.

25. PAULA LG, et al. Partograma: procedimento essencial em obstetrícia [Internet]. Brasília: Portal Médico; 2001 [cited 2011 nov 22].

26. PEREIRA WR. Poder, violência e dominação simbólicas em um serviço público de saúde que atende a mulheres em situação de gestação, parto e puerpério. [tese]. Ribeirão Preto: Escola de Enfermagem de Ribeirão Preto da Universidade de São Paulo; 2000.

27. PORTO AMF, et al. Assistência ao primeiro período do trabalho de parto baseada em evidências. Femina. Outubro, 2010, 38(10).

28. REZENDE J. Obstetrícia fundamental. Rio de Janeiro: Guanabara-Koogan, 1997.

29. ROCHA IMS, et al. O Partograma como instrumento de análise da assistência ao parto. Revista da Escola de Enfermagem da USP, São Paulo, 2009, 43(04).

30. ROSA. Eduardo. Estudo terminológico na área de medicina obstétrica. 2014.

31. SILVA LB. Estudo terminológico na área de medicina obstétrica: problemática da comunicação médico - paciente. 2015.

32. SOUZA GF. Instrumento de boas práticas de enfermagem em hemoterapia na unidade de terapia intensiva: uma construção coletiva. [dissertação]. Florianópolis (SC): Universidade Federal de Santa Catarina, Programa de PósGraduação em Enfermagem; 2012.

33. TEIXEIRA LRM. Indução do Trabalho de Parto: métodos farmacológicos. Faculdade de Medicina Universitária do Porto. Abril, 2010.

34. TRAVERZIM MAS, NOVARETTI MCZ. Estudo dos eventos adversos em obstetrícia em um hospital público do município de São Paulo. In: III SIMPÓSIO INTERNACIONAL DE GESTÃO DE PROJETOS/ II SIMPÓSIO, 2014.

35. VASCONCELOS KL, et al. Partograma: Instrumento para segurança na assistência obstétrica. Revista de Enfermagem UFPE On line, Recife, fev. 2013, 7(2): 619-24. 